\title{
Evaluation de la toxicité de cinq plantes antiasthmatiques de la médecine traditionnelle ivoirienne
}

\author{
Amenan Geneviève IRIE-N'GUESSAN ${ }^{1-4 *}$, Brou Jérôme KABLAN ${ }^{4}$, N'doua Gisèle \\ KOUAKOU-SIRANSY ${ }^{4}$, Véronique LEBLAIS ${ }^{2-3}$ et Pierre CHAMPY ${ }^{1-2}$ \\ * Laboratoire de pharmacologie et physiologie, UFR Sciences Pharmaceutiques et Biologiques, Université \\ Cocody-Abidjan 01 BP V 34, République de Côte d'Ivoire. \\ ${ }^{1}$ Chimie des substances naturelles et chimiothérapie antiparasitaire, CNRS UMR 8076 BioCIS, Châtenay- \\ Malabry, France. \\ ${ }^{2}$ Université Paris-Sud 11, Faculté de Pharmacie, Châtenay-Malabry, France. \\ ${ }^{3}$ INSERM UMR-S 769, Châtenay-Malabry, France. \\ ${ }^{4}$ Laboratoire de pharmacologie, pharmacie clinique, thérapeutique et physiologie, UFR Sciences \\ Pharmaceutiques et Biologiques, Université Cocody-Abidjan, République de Côte d'Ivoire. \\ *Auteur correspondant, E-mail: jemigrace@gmail.com, Tel: +225-07988157
}

\section{RESUME}

Les feuilles de Boerhavia diffusa Linn. Sp. (Nyctaginaceae), Baphia nitida Lodd., Cassia occidentalis Linn. Sp., Desmodium adscendens (Sw.) DC. et l'écorce de racines de Dichrostachys cinerea (L.) Wight et Arn. (Fabaceae) sont traditionnellement utilisées pour traiter l'asthme en Côte d'Ivoire. Les propriétés antispasmodiques de quatre extraits totaux (décocté, hydro-alcoolique, méthanolique et chlorométhylénique) de ces substances végétales sur la musculature lisse du tractus respiratoire ont précédemment été montrées sur la trachée isolée de souris, justifiant certainement leur usage antiasthmatique en médecine traditionnelle. La présente investigation a consisté en l'étude de la toxicité de ces extraits totaux de plantes. Les tests de cytotoxicité réalisés in vitro sur cellules $\mathrm{KB}$ en culture ainsi que les tests in vivo chez la souris avec des doses similaires aux concentrations testées sur la trachée isolée n'ont pas mis d'effets toxiques en évidence à ces doses, suggérant une certaine sécurité d'emploi de ces substances végétales dans le traitement traditionnel de l'asthme.

(C) 2011 International Formulae Group. All rights reserved.

Mot clés : Boerhavia diffusa, Baphia nitida, Cassia occidentalis, Desmodium adscendens, Dichrostachys cinerea, toxicité.

\section{INTRODUCTION}

Les feuilles de Boerhavia diffusa Linn. Sp. (Nyctaginaceae), Baphia nitida Lodd., Cassia occidentalis Linn. Sp., Desmodium adscendens (Sw.) DC. et l'écorce de racines de Dichrostachys cinerea (L.) Wight et Arn.
(Fabaceae) sont traditionnellement préparées sous forme de décoctions, seules ou en association avec d'autres plantes, pour traiter l'asthme et autres affections respiratoires en Côte d'Ivoire (Adjanohoun et Aké-Assi, 1979). Des tests ont montré l'effet anti- 
inflammatoire et antioxydant de $B$. diffusa, $B$. nitida et $C$. occidentalis (Kouakou-Siransy et al., 2010), l'effet antispasmodique de $D$. adcendens (Addy, 1989) et de D. cinerea (Aworet-Samseny et al., 2011). Précédemment, nous avons mis en évidence l'effet antispasmodique d'extraits totaux de polarités différentes de ces substances végétales sur la trachée isolée de souris. La présente investigation étudie la toxicité de ces substances végétales in vitro en culture cellulaire et in vivo chez la souris pour évaluer leur sécurité d'emploi en médecine traditionnelle.

\section{MATERIEL ET METHODES Matériel végétal}

Les feuilles matures de $B$. diffusa, $B$. nitida, D. adscendens et $C$. occidentalis ont été récoltées en Janvier 2009 au sud de la Côte d'Ivoire, dans le village de Yakassé-Mé (région de l'Agnéby), pendant que les racines de $D$. cinerea l'ont été dans la même période au sud-est de la Côte d'Ivoire, dans des buissons près de Grand-Bassam (région des lagunes). Les plantes ont été identifiées par un taxonomiste du Centre National de Floristique (Abidjan-Côte d'Ivoire) et des spécimens y ont été déposés. Ecorces et feuilles ont été lavées, séchées sous air conditionné pendant deux semaines, pulvérisées et quatre extraits totaux ont été préparés (décocté, hydroalcoolique, méthanolique et chlorométhylénique).

\section{La cytotoxicité in vitro}

La mesure de la cytotoxicité a été effectuée sur cellules de tumeur du nasopharynx humain (cellules KB ATCC CCL-17) mises en culture dans des boîtes de $75 \mathrm{~cm}^{3}$ à l'étuve pendant quatre jours à $37^{\circ} \mathrm{C}$ dans une atmosphère à $95 \%$ d'oxygène et $5 \%$ dioxyde de carbone. Ces cellules ont été distribuées dans des plaques de 96 puits, à raison de $2 \mathrm{ml}$ de suspension contenant 75000 cellules dans chaque puits. Après 24 h, $2 \mu \mathrm{l}$ de chaque concentration d'extrait de plante (10 à $10^{-3} \mu \mathrm{g} / \mathrm{ml}$ d'extrait de plante dans le DMSO) ont été déposés en double ou triple ainsi que les témoins de solvant (DMSO = témoin négatif) et de Taxotère ${ }^{\circledR}$ (docétaxel = témoin positif). Au bout de 72 heures, l'inhibition éventuelle de la prolifération cellulaire a été évaluée en comparaison avec les cultures témoins négatifs par ajout de $40 \mu \mathrm{l}$ de MTS (sel de tétrazolium métabolisé par les cellules viables et métaboliquement actives en un formazan coloré qui absorbe à $490 \mathrm{~nm}$ ). Après 2h d'incubation, la densité optique (DO) de chaque puits a été mesurée au spectrophotomètre. Le pourcentage d'inhibition a été ainsi déterminé (le colorant ne pénétrant que dans les cellules vivantes, la coloration est d'autant plus faible que l'extrait de plante est cytotoxique par inhibition de la prolifération des cellules $\mathrm{KB}$ ). Le témoin Taxotère $^{\circledR}$ a été utilisé pour évaluer le degré d'inhibition de la croissance des cellules.

\section{La toxicité in vivo}

La détermination de cette toxicité s'est faite selon la méthode de Lietchfiel et Wilcoxon (1949) sur des souris de souche Swiss pesant 20-30 g et fournies par le centre d'élevage de l'Institut Pasteur d'Adiopodoumé (Côte d'Ivoire). 4 lots de 10 souris de poids homogène $(25 \pm 5 \mathrm{~g})$ dont 1 lot témoin ont été constitués pour chaque extrait de plante. Les souris ont été mises à jeun de nourriture $18 \mathrm{~h}$ avant les tests. 3 concentrations d'extraits ont été testées : 1 $\mathrm{mg} / \mathrm{ml}, 10 \mathrm{mg} / \mathrm{ml}$ et $100 \mathrm{mg} / \mathrm{ml}$. Les souris de chaque lot-test ont reçu une concentration donnée par gavage à raison de $10 \mathrm{ml} / \mathrm{kg}$ de poids corporel tandis que les souris du lot témoin n'ont reçu que le solvant de dilution (l'eau distillée) à raison de $10 \mathrm{ml} / \mathrm{kg}$ de poids corporel. $2 \mathrm{~h}$ plus tard, les souris ont eu droit à une alimentation normale. Le taux de mortalité au sein de chaque lot a été enregistré au bout de 72 heures. 


\section{RESULTATS ET DISCUSSION}

Sur les 20 extraits totaux testés sur des cellules $\mathrm{KB}$ en culture, seuls 2 , à savoir les extraits chlorométhyléniques de $B$. nitida et de C. occidentalis ont donné un fort pourcentage d'inhibition de la croissance cellulaire à 10 $\mu \mathrm{g} / \mathrm{ml}$, à raison de $100 \%$ et $89 \%$ respectivement. Toutefois, ces 2 extraits n'ont montré aucune activité cytotoxique à $1 \mu \mathrm{g} / \mathrm{ml}$ (Tableau 1).

L'étude la toxicité in vivo, 72 heures après l'administration par voie orale des extraits des substances végétales aux différents lots de souris, n'a enregistré aucun mort. Les plus fortes concentrations d'extraits totaux ayant été administrées étant de 100 $\mathrm{mg} / \mathrm{ml}$ à raison de $10 \mathrm{ml} / \mathrm{kg}$ de poids corporel, soit des doses de $1000 \mathrm{mg} / \mathrm{kg}$ de poids corporel, les Doses Minimales Toxiques (DMT) des extraits de ces 5 substances végétales sont donc supérieures à $1000 \mathrm{mg} / \mathrm{kg}$ de poids corporel. Cette absence de toxicité aiguë aux doses correspondant aux concentrations testées in vitro est conforme à l'observation que nous avons faite au cours des expérimentations sur la trachée isolée. En effet, après chaque test, les anneaux de trachée étaient lavés et au bout d'une période de repos d'une heure, les anneaux étaient précontractés avec le carbachol pour vérifier leur intégrité fonctionnelle: les anneaux se contractaient exactement comme avant qu'ils n'aient été mis en contact avec les différents extraits de plantes, suggérant que les différentes substances végétales ne causaient pas de lésion tissulaire.

Les résultats obtenus au cours de notre étude corroborent ceux d'autres auteurs sur les mêmes plantes. Ainsi, il a été montré que l'extrait aqueux des feuilles de B. diffusa n'était pas toxique chez le rat aux doses de 500,1000 et $2000 \mathrm{mg} / \mathrm{kg}$ de poids corporel administrées per os (Orisakwe et al., 2003). De même, il n'a pas été observé de toxicité aiguë avec l'extrait alcoolique des feuilles de B. nitida chez le rat (Onwukaeme et Lot, 1991). Concernant C. occidentalis, si ses fruits sont réputés très toxiques (Barbosa-Ferreira et al., 2005), les parties aériennes n'ont montré chez les rattes gestantes d'autres signes de toxicité en dehors de la mort de certains fœetus aux doses de 250 et $500 \mathrm{mg} / \mathrm{kg}$ de poids corporel (Aragão et al., 2009). Pour ce qui est de $D$. adscendens, une expertise toxicologique, effectuée en France, confirme la non-toxicité de la plante (C.I.T., 1989). Par contre, l'extrait alcoolique des racines de $D$ cinerea aurait montré des effets neurotoxiques à 100,200 et $400 \mathrm{mg} / \mathrm{kg}$ de poids corporel en administration orale à des souris Swiss (Ramya-Kuber, 2009).

Tableau 1 : Pourcentage d'inhibition de la prolifération cellulaire des extraits à $10 \mu \mathrm{g} / \mathrm{ml}$ et/ou 1 $\mu \mathrm{g} / \mathrm{ml}$.

\begin{tabular}{lccccc}
\hline & \multicolumn{5}{c}{ Pourcentage d'inhibition de la prolifération cellulaire (\%) } \\
\cline { 2 - 6 } & B. diffusa & B. nitida & C. occidentalis & D. adscendens & D. cinerea \\
\hline $\mathrm{H}_{2} \mathrm{O}$ & 0 & 0 & 0 & 6 & 0 \\
$\mathrm{H}_{2} \mathrm{O} / \mathrm{EtOH}$ & 0 & 0 & 0 & 6 & 1 \\
$\mathrm{MeOH}$ & 0 & 12 & 0 & 0 & 0 \\
$\mathrm{CH}_{2} \mathrm{Cl}_{2}$ & 11 & $\mathbf{1 0 0 / 0}$ & $\mathbf{8 9 / 0}$ & 3 & 0 \\
\hline
\end{tabular}

La deuxième valeur correspond au pourcentage d'inhibition à $1 \mu \mathrm{g} / \mathrm{ml}$.

$\mathrm{H}_{2} \mathrm{O}=$ décocté ; $\mathrm{H}_{2} \mathrm{O} / \mathrm{EtOH}=$ extrait hydro-alcoolique ; $\mathrm{MeOH}=$ extrait méthanolique ;

$\mathrm{CH}_{2} \mathrm{Cl}_{2}=$ extrait chlorométhylénique 


\section{Conclusion}

Il ressort de l'étude de la cytotoxicité et de la toxicité aiguë, aux doses conformes aux concentrations des substances végétales testées ayant montré un effet antispasmodique sur la trachée isolée de souris, que ces doses ne sont pas toxiques. L'usage de ces substances végétales en médecine traditionnelle pour traiter l'asthme bénéficierait donc d'une sécurité d'emploi pouvant être mis à profit pour le développement de phytomédicaments à moindre coût dans les pays en développement dont la Côte d'Ivoire où les prévalences de l'asthme sont en recrudescence avec toutefois un pouvoir d'achat faible.

\section{REMERCIEMENTS}

Les auteurs sont reconnaissants de l'assistance technique de Laurent Aké-Assi (Centre National de Floristique d'Abidjan) pour l'identification botanique, et de Thierry Cresteil (Institut de Chimie des Substances Naturelles CNRS-UPR2301, Paris) pour les tests de cytotoxicité.

\section{REFERENCES}

Addy ME. 1989. Several chromatographically distinct fractions of Desmodium adscendens inhibit smooth muscle contractions. Int. J. Crude Drug Res., 27(2): 81-91.

Adjanohoun EJ, Aké-Assi L. 1979. Contribution au Recensement des Plantes Médicinales de Côte d'Ivoire. Centre National de Floristique : Abidjan ; 238 p.

Aragão TP, Lyra MMA, Silva MGB, Andrade BA, Ferreira PA, Ortega LF, da Silva SD, da Silva JCP, Fraga MCCA, Wanderley AG, Lafayette SSL. 2009. Toxicological reproductive study of Cassia occidentalis L. in female Wistar rats. $J$. Ethnopharmacol., 123: 163-166.
Aworet-Samseny RR, Souza A, Kpahé F, Konaté K, Datté JY. 2011. Dichrostachys cinerea (L.) Wight et Arn (Mimosaceae) hydro-alcoholic extract action on the contractility of tracheal smooth muscle isolated from guinea-pig. BMC Compl. Altern. Med., 11: 23.

Barbosa-Ferreira M, Dagli MLZ, Maiorka PC, Górniak SL. 2005. Sub-acute intoxication by Senna occidentalis seeds in rats. Food Chem. Toxicol., 43: 497-503. C.I.T. 1989.

Desmodium adscendens -."Evaluation de la toxicité aiguë par voie orale chez le rat". Centre International de Toxicologie Evreux, France $\mathrm{n}^{\circ} 5374$ TAR (18/10/1989). [tiré de http://www. oronalia.com/fichier/DESMODIUMADS CENDENS32.pdf ]

Kouakou-Siransy G, Sahpaz S, Irie-Nguessan G, Datte YJ, Kablan J, Gressier B, Bailleul F. 2010. Oxygen species scavenger activities and phenolic contents of four West African plants. Food Chem., 118(2): 430-435.

Lichtfield JF, Wilcoxon FA. 1949. A simplified method of evaluation doseseffects experiments. J. Pharmacol. Exper. Therap., 95: 99-113.

Onwukaeme ND, Lot TY. 1991. A pharmacological evaluatio $\mathrm{n}$ of Baphia nitida Lodd (Leguminosae) ethanolic extract on rats and mice. Phytother. Res., 5(6): 254-256.

Orisakwe OE, Afonne O J, Chude MA, Obi E, Dioka CE. 2003. Sub-chronic Toxicity Studies of the Aqueous Extract of Boerhavia diffusa Leaves. J. Health Sci., 49(6): 444-447.

Ramya-Kuber B, SanthRani T. 2009. Evaluation of Neuropharmacological Effects of Dichrostachys cinerea Root. Int. J. Pharmaceutical Sci. Nanotechnol., 1(4): $367-374$. 